\title{
Hybrid Spread Spectrum based Smart Meter Network using Fast Frequency Hopping
}

\author{
Aditya Kothari \\ Master of Engineering \\ E \& TC Department \\ SGSITS Indore, M.P.
}

\author{
Manish Panchal \\ Astt. Professor \\ E \& TC Department \\ SGSITS Indore, M.P.
}

\author{
Rekha Jain \\ Astt. Professor \\ E \& TC Department \\ SGSITS Indore, M.P.
}

\begin{abstract}
Smart metes used in electric grids need a dedicated network that should be highly reliable \& cost effective. Various techniques like $3 \mathrm{G}$ cellular have been proposed to improve efficiency of this smart grid electric meter network. For distribution of proper information in smart grid system Hybrid Spread Spectrum using slow frequency technology is also better choice. To improve the performance of this network in the terms of throughput and number of smart meter per data aggregation point (DAP), we have proposed HSS-FFH (to implement AMI) method. These techniques give better result in terms of coverage high density population area $\&$ interference immunity.
\end{abstract}

\section{Keywords}

AMI, DAP, HSS, Fast Frequency Hopping, Slow Frequency Hopping

\section{INTRODUCTION}

The Electric grid, which uses smart technology, so as to collect information about power consumption of customer, in an automated fashion is termed as Smart Grid. This results in improved efficiency, reliability and sustainability of production and distribution of electricity. Objective of smart grid is to improve the generation transmission and distribution (GTD). At the distribution site, one device is required to communicate information for billing customers and operating their electric systems and here smart meter performs the role significantly. The combination of the electronic meters with two-way communication technology for information, monitor, and control is commonly referred to as Advanced Metering infrastructure (AMI) [fig 1].

Spread-spectrum modulation techniques have been adopted for many current and future military communication systems to accommodate high data rates with high link integrity, even in the presence of significant interfering signals. A more recent synergistic combination is a direct-sequence spreadspectrum (DSSS) signaling with the use of integrally coordinated frequency hopping (FH) and/or time-hopping (TH) modulation, generically dubbed hybrid spread-spectrum (HSS). A highly useful form of this transmission scheme for many types of command, control, and sensing applications is the specific code-related combination of standard DSSS modulation with "Fast" Frequency Hopping (FFH), wherein multiple frequency hops occur within a single data-bit time. The technique can be used in smart metering system.

The following are the main contributions of this paper:

1) Some inefficiencies of using $3 \mathrm{G} / 4 \mathrm{G}$ technologies for distribution side smart grid applications have been observed. The issues are discussed in detail.
2) Furthermore use of HSS-SFH technology, modification, and comparison with HSS-FFH has been performed.

3 ) Implementation of the proposed HSS-FFH ( PHY and MAC layer) in the packet-based network is also discussed.

\section{PRECURSORY}

\subsection{AMI NETWORK}

Previous systems, which utilized one-way communications to collect meter data, were referred to as Automated Meter Reading (AMR) Systems [4]. AMI has developed over time, from its roots as a metering reading substitute (AMR) to today's two-way communication and data system. Advance Metering Infrastructure is a technology where the traditional electricity meters are going to be replaced by smart meters at the customer's site, which give the utilities the ability to monitor and collect information related to the amount of electricity consumed by the customer [7]. As shown in Fig 1, we assume a two layered communication architecture for the AMI network. The utility office collects the data from the smart meters, and the utility pole Data Aggregation Point (DAP) acts as a relay between the smart meter and the utility office. The number of smart meters that a DAP handles can vary from as few as 100 to as many as 50,000 , depends on requirement. The data collection time interval could be 15 minutes or 30 minutes or 1 hour, depends on number of smart meters and latency of technology used [4].

\subsection{G/4G ISSUES}

The main issues of using the current state of the art $3 \mathrm{G} / 4 \mathrm{G}$ cellular technologies for smart grid applications are as follows:

Code Division Multiple Access (CDMA) and Wideband Code Division Multiple Access (WCDMA) both employ a Transmission Control Protocol/Internet Protocol (TCP/IP) based data protocol stack [8]. Compared with smart phone applications, the data transfer involving smart grid applications is very low (i.e., hundreds of Bytes) [10]. Even with the use of RObust Header Compression (ROHC), the percentage of TCP/IP overhead for smart grid data is very high because the TCP/IP overhead not only comes from the header size but also from the way TCP operates $[6,9]$. TCP uses a 3-way handshake for connection establishment and connection termination between a source and a destination. As a result, the relative overhead (overhead with respect to payload) of TCP/IP packets is around 5\% to $10 \%$ [8]. Taking the above factors into consideration, it appears that the TCP/IP based 3G cellular technology is not an appropriate choice for smart grid applications. 
In Fig 1, assuming that there is a database at the DAP, the wireless portion of the network can be considered as a smart metering sensor network. No wireless sensor networks use TCP/IP based stack as it results in data overhead and latency. Therefore from the utilities perspective, it is worth considering the idea of designing a custom AMI network rather than using an already existing $3 \mathrm{G} / 4 \mathrm{G}$ network.

In order to overcome the near-far problem and combat Doppler shift, 3G cellular technology employs closed loop power control. Power control is a continuous process and it operates at a frequency of $1500 \mathrm{~Hz}$ [11] i.e. every $0.66 \mathrm{msec}$ Base Station (BS) performs a computation related to the transmit power of an active Mobile Station (MS) in its cell and sends power control information to the MS to decrease/increase the transmit power by $\delta \mathrm{dB}$ during transmission of next frame. In the case of AMI, most of the smart grid devices are stationary. Hence there is no chance of smart meters experiencing Doppler shift because of mobility but there is a slight possibility of Doppler Effect due to changes in environment. Therefore, like CDMA, AMI network may not require faster power control. A closed power control that operates at a low frequency $(<100 \mathrm{~Hz})$ should be sufficient for this task.

As far as Long Term Evolution (LTE-4G) is concern it relies on Orthogonal Frequency Division Multiple Access (OFDMA) and Multiple Input Multiple Output (MIMO) and targeted at achieving high data rates around $100 \mathrm{Mbps}$. smart grid application data rate requirements are less than 250 kbps. Compared to LTE, spread spectrum based technologies excel at these data rates and also exhibit well multiple access properties. Therefore designing a spread

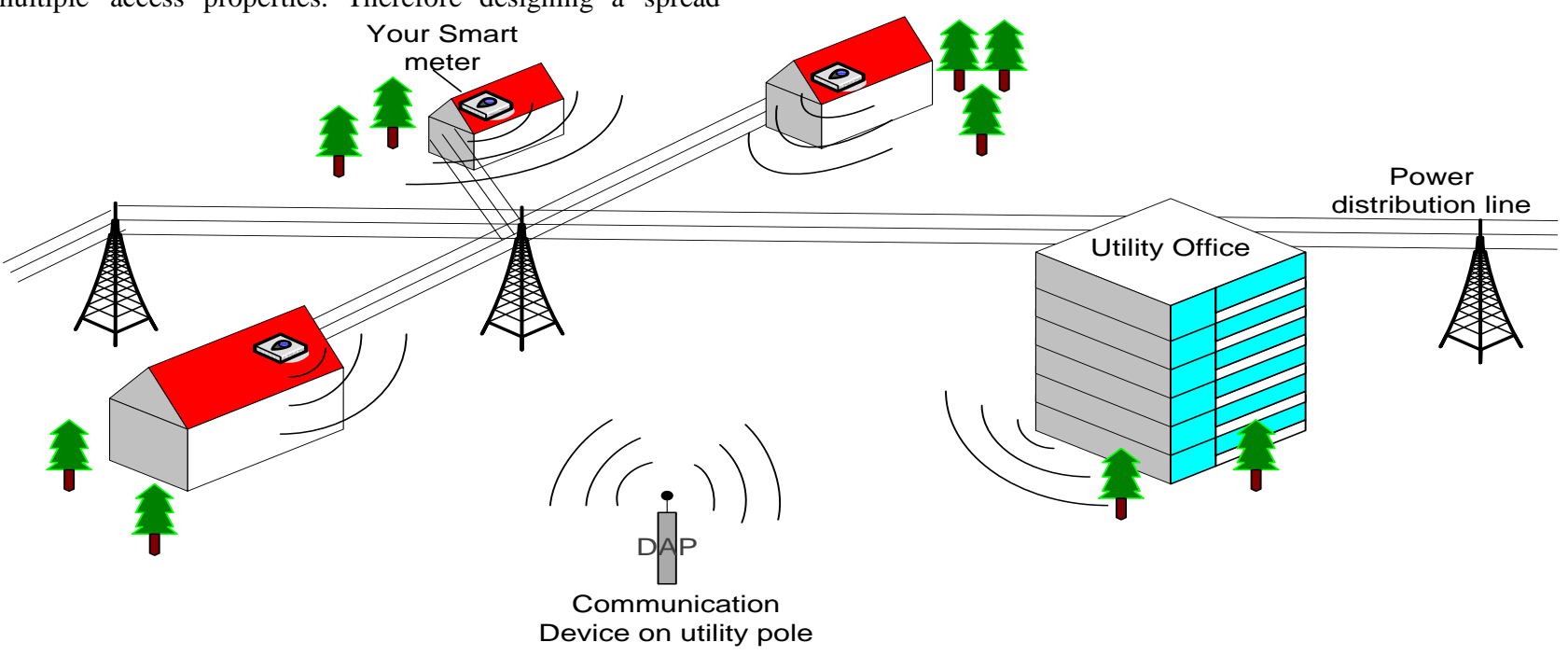

spectrum based network is more appropriate for smart grid applications rather than using OFDMA based 4G technologies.

\subsection{HSS-SFH ISSUES}

Multiple access schemes are required to support a high number of smart meters. CDMA is selected as the multiple access schemes with hybrid spread spectrum (HSS) as the physical layer technique. It uses Slow Frequency Hopping (SFH) for operation. Main reason for the selection of CDMA for the AMI network design is that the CDMA outperforms TDMA and FDMA in noise, interference and jamming environments.

\subsection{HSS-FFH BASED AMI}

The proposed scheme is Hybrid Spread Spectrum-fast frequency hopping based AMI. As the name implies, the technique uses Fast Frequency Hopping Instead of previous technique of using Slow Frequency Hopping. In HSS-SFH the frequency is hopped every 100 bits (i.e. Th $=100$ bits). While in FFH the frequency is hopped more than once in single bit per bit duration (i.e. $\mathrm{Th}=0.25$ bit duration). The FFH ratio is taken to be $4: 1$ for ease of understanding. One can increase this ratio for more security. We use Time Division Multiple Access (TDMA) multiple access scheme in the place of CDMA. Main reason of selecting TDMA is, FFH make it noise and interference immune. FFH is used in military application to increase security. Use of FFH makes the smart meter data more secure, as frequency tracing is difficult duration. So in HSS-FFH scheme, 4 frequencies are hopped

\section{Fig 1: Advance Metering Infrastructure (AMI) [7]}

\section{NS2 SIMULATION ISSUES}

There are many issues in implementing a new wireless protocol in NS-2. The current implementation of wireless PHY and MAC layer of NS-2 relies heavily on IEEE 802.11 and IEEE 802.15.4.

As already discussed, we use TDMA access scheme in HSSFFH technique. First of all, the channel parameters settings are done according to the requirement, which includes MAC protocol which is set to be Wireless/TDMA. Furthermore UDP Packet size is taken to be 210 bytes. The Queue limit is set to be 150 , which means $150 * 210=31500$ bytes. Using robust header compression reduces the size of header to 4 byes instead of 40 bytes. The overhead for one packet transfer is 206 bytes of data +4 byte of header i.e. Less than $2 \%$ of overheads and rest $98 \%$ of data is transferred. The overhead can be minimized by increasing packet size. After these the routing protocol used is Adhoc On-demand Distance Vector (AODV). The RF exposure limits, set by the Fedral Communication Commission (FCC) for Smart Meters are rated at the frequencies they use to communicate is $915 \mathrm{MHz}$ $601 \mu \mathrm{W} / \mathrm{cm} 2$ avg. and $2.4-100 \mathrm{GHz} 201000 \mu \mathrm{W} / \mathrm{cm} 2$. The power transmitted from the smart meter is taken to be 0.031622777 Watts, and receiver threshold is set to 
be $5.82587 \mathrm{e}^{-09}$ Watts. The Smart Meters use low power transmitters, hence the proposed scheme falls under FCC limit [4]. It produces relatively weak RF signals. These Values of wireless simulation parameters are based on Wu Xiucho's technical report [12].

\subsection{RESULTS}

The three parameters which calculated by NS-2 are most important; Average Throughput, End to End delay, and Normalized Routing Load (Refer Table: 1)

Table 1: NS-2 Parameter Results

\begin{tabular}{|c|c|c|c|}
\hline $\begin{array}{c}\text { No. of } \\
\text { nodes }\end{array}$ & $\begin{array}{c}\text { Throughput } \\
(\mathbf{K b p s})\end{array}$ & $\begin{array}{c}\text { End to end delay } \\
(\mathbf{m s})\end{array}$ & $\begin{array}{c}\text { Normalized } \\
\text { routing load }\end{array}$ \\
\hline 2 & 99.44 & 927.874 & 0.0134228 \\
\hline 4 & 108.15 & 1885.21 & 0.0134228 \\
\hline 6 & 143.05 & 2679.58 & 0.0143062 \\
\hline 8 & 149.70 & 2963.02 & 0.0629275 \\
\hline 13 & 150.27 & 4621.5 & 0.113736 \\
\hline 23 & 161.14 & 9083.83 & 0.18146 \\
\hline 31 & 134.67 & 10381.1 & 0.331344 \\
\hline
\end{tabular}

The Graph 1 shows comparison of HSS-SFH with HHS-FFH. $\mathrm{X}$-axis specifies offered load (node) and $\mathrm{Y}$-axis specifies throughput in Kbps. The throughput is becoming low for less Node value and gradually increasing to peak value of $161.14 \mathrm{Kbps}$ and then settle down to around $120 \mathrm{Kbps}$. This nature of curve is due to AODV control frames. This shows far good results compared to HSS-SFH technique, which have 40Kbps maximum throughput.

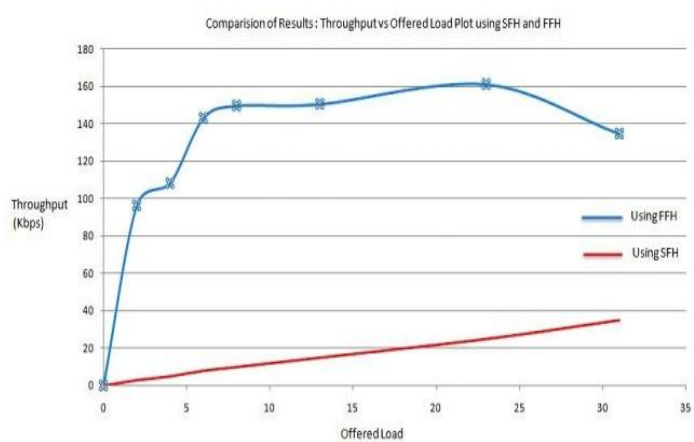

\section{Graph 1: Comparison of HSS-SFH and HSS-FFH} (Throughput)

This is obvious from simulation results that HSS-FFH is showing batter throughput, as the packet drop is less due to FFH.

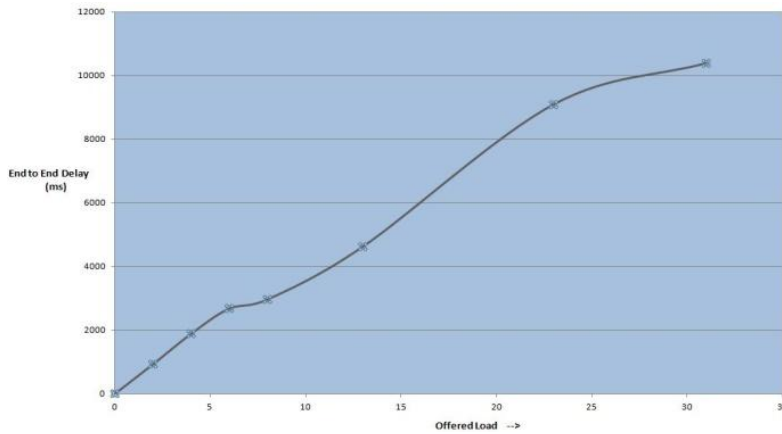

Graph 2: Offered Load vs End to End Delay for HHSFFH

The $\mathrm{X}$-axis and $\mathrm{Y}$-axis in Graph 2 specifies offered load and end to end delay in milliseconds. The curve is increasing exponentially. The delay seems to be quite large. It is because of Time Scheduling Scheme. In Time Scheduling Scheme each smart meter transmits their data one by one, to transmit the power consumption data. The End to End delay consist the time delay of $200 \mathrm{~ms}$ for each node, to avoid interference. For HSS-SFH technology End to End delay is $1300 \mathrm{~ms}$ at 8 nodes and $4000 \mathrm{~ms}$ at 30 nodes. This is comparable with HHS-FFH technology by including $200 \mathrm{~ms}$ delay at each node.

Average Throughput can be calculated With $4^{\text {th }}$ Order Polynomial Equation. The equation is valid for node values up to 30 at each DAP.

$\mathrm{T}_{\mathrm{h}}=-0.001 \eta^{4}+0.093 \eta^{3}-2.329 \eta^{2}+25.46 \eta+49.58-(1)$

Same as above the Average End to End Delay calculation with $3^{\text {rd }}$ Order Polynomial Equation is written below.

$E_{d}=-0.453 \eta^{3}+19.40 \eta^{2}+143.1 \eta+848.3$

Where:

- $\quad \eta=$ no of nodes

- $\mathrm{T}_{\mathrm{h}}=$ Throughput

- $\mathrm{E}_{\mathrm{d}}=$ Average End To End Delay

The third parameter is the normalized routing load, which is the number of routing packets transmitted per data packet sent to destination. The results are shown in Table 1 last column. The NRL increases as the nodes value increases. It is acceptable till node value of 15 at a DAP. It shows batter results as, in HSS-SFH, one DAP can handle 8 nodes. The result shows that use of HSS-FFH technique in AMI improve bandwidth efficiency, latency, and reliability.

\section{CONCLUSION}

The use of HSS-FFH technology in AMI network is very much effective in achieving our goals. By the use of time scheduling number of smart meter users are increasing therefore best suited for high population density area. Interference immunity increases, because the frequency is hoped more than once in single bit duration. It is showing Better Throughput, latency and NRL as compared to the previous concept of applying HSS-SFH. Hence it is concluded that HSS-FFH technology is better option for smart grid distribution side applications, as compared with 3G, 4G, HSSSFH and other technologies. 


\section{ACKNOWLEDGMENTS}

Our thanks to the members of the Department of Electronics and Telecommunication for their guidance and suggestions, especially Dr. P. D. Vyavahare, Head of Department, for his caring attitude and encouragement. We appreciate the support of Dr. S. S. Bhadauria, Director SGSITS, Indore for motivating us to research, by providing the academic facilities in this institute.

\section{REFERENCES}

[1] A. Nesbitt, B. Stewart, S. McMeekin, S. Conner, J. Gamio, K. Liebech- Lien, H. Kristiansen, and S. Krakenes, "A novel approach to high voltage substation surveillance using radio frequency interference measurement," in Electrical Insulation Conference, 2009. EIC 2009. IEEE, 31 2009- june 3 2009, pp. 159 163.

[2] M. Olama, X. Ma, T. Kuruganti, S. Smith, and S. Djouadi, "Hybrid ds/ffh spread-spectrum: A robust, secure transmission technique for communication in harsh environments," in MILITARY COMMUNICATIONS CONFERENCE, 2011 - MILCOM 2011, nov. 2011, pp. $2136-2141$.

[3] E. Geraniotis, "Coherent hybrid ds-sfh spread-spectrum multiple-access communications," Selected Areas in Communications, IEEE Journal on, vol. 3, no. 5, pp. 695 - 705, sep 1985.

[4] EEI-AEIC-UTC White Paper, "Smart Meter and Smart meter Systems : A Metering Industry Perspective", March 2011.
[5] Marc Greis, "NS2 Tutorial Documentation" maintained and being expanded by VINT group.

[6] Frank Fitzek, Stefan Hendrata, Patrick Seeling, and Martin Reisslein,"Header Compression Schemes for Wireless Internet Access" Book First Edition, pp. 05-25, July 2003.

[7] Shravan Garlapati, Haris I. Volos, Teja Kuruganti, Michael R. Buehrer, Jeffrey H. Reed, "PHY and MAC Layer Design of Hybrid Spread Spectrum Based Smart Meter Network", in Smart Grid Communication IEEE International Conference on, Oct 2012, pp. 2136-2141.

[8] J. D. Cavanaugh, "Protocol overhead in ip/atm networks," 1994.

[9] F. Frank, H. Stefan, S. Patrick, and M. Reisslein. (2003) Header Compression schemes for wireless internet access. [Online]. Available: www.3g4g.co.uk/Other/ROHC/Whitepapers/rohc book.pdf.

[10] H.-H. Chen, The next generation CDMA technolgies. West Sussex, England: John Wiley \& Sons Ltd, 2007.

[11] Marc Greis, "NS2 Tutorial Documentation" maintained and being expanded by VINT group.

[12] Xiucho.W and Ananda.A.L, Link Characteristics Estimation for 802.11 DCF Based WLAN, Proc 29th Annual IEEE International conference on local computer network, Washington, DC, 2004, 302-309. 